\title{
Exotic baryons as a hadronic molecule in the heavy quark region
}

\author{
Yasuhiro Yamaguchi ${ }^{1, \star}$ \\ ${ }^{1}$ Istituto Nazionale di Fisica Nucleare (INFN), Sezione di Genova, via Dodecaneso 33, 16146 Genova, Italy
}

\begin{abstract}
We study the hadronic molecules formed by the coupled-channel system of $\bar{D}^{(*)} \Lambda_{\mathrm{c}}$ and $\bar{D}^{(*)} \Sigma_{\mathrm{c}}^{(*)}$, inspired by the two hidden-charm pentaquark states observed by LHCb collaborations in 2015. In these molecules, the coupled channels of $\bar{D}^{(*)} \Sigma_{\mathrm{c}}^{(*)}$ are important because the thresholds of these channels are approximately degenerated by the heavy quark spin symmetry. In addition, we consider the coupling to the $\bar{D}^{(*)} \Lambda_{\mathrm{c}}$ channel whose thresholds are close to the $\bar{D}^{(*)} \Sigma_{\mathrm{c}}^{(*)}$ thresholds, and the coupling to the state with $\ell \neq$ 0 mixed by the tensor force. By solving the coupled-channel Schrödinger equations with the one meson exchange potentials, we study the hidden-charm hadronic molecules with $I\left(J^{P}\right)=1 / 2\left(3 / 2^{ \pm}\right)$and $1 / 2\left(5 / 2^{ \pm}\right)$. We conclude that the $J^{P}$ assignment of the observed pentaquarks is $3 / 2^{+}$for $P_{\mathrm{c}}^{+}(4380)$ and $5 / 2^{-}$for $P_{\mathrm{c}}^{+}(4450)$, which is agreement with the results of the LHCb analysis. In addition, we give predictions for other $J^{P}=3 / 2^{ \pm}$states.
\end{abstract}

\section{Introduction}

In 2015, LHCb collaborations observed the two hidden-charm pentaquarks $P_{\mathrm{c}}^{+}(4380)$ and $P_{\mathrm{c}}^{+}(4450)$ in $\Lambda_{\mathrm{b}}^{0} \rightarrow J / \psi K^{-} p$ decay [1]. The minimal quark content of them is considered to be $c \bar{c} u u d$. The reported masses and widths are $(M, \Gamma)=(4380 \pm 8 \pm 29,205 \pm 18 \pm 86) \mathrm{MeV}$ and $(4449.8 \pm 1.7 \pm 2.5,39 \pm 5 \pm 19)$ $\mathrm{MeV}$, respectively. In Ref. [1], the $J^{P}$ of $\left(P_{\mathrm{c}}^{+}(4380), P_{\mathrm{c}}^{+}(4450)\right)$ is $\left(3 / 2^{-}, 5 / 2^{+}\right)$. However, $\left(3 / 2^{+}, 5 / 2^{-}\right)$, and $\left(5 / 2^{+}, 3 / 2^{-}\right)$are also acceptable. There have been lively discussions about the structure of the pentaquarks, such as the compact pentaquark [2-5] and the hadronic molecule [6-14]. On the other hand, the threshold enhancement by the anomalous triangle singularity is discussed in Refs. [16-18].

Hadronic molecules are expected as the structure of the exotic states near the thresholds. In the strangeness sector, $\Lambda(1405)$ has been discussed as a meson-baryon $(\bar{K} N$ and $\pi \Sigma)$ molecule [19-21]. A lot of exotic states have been found near the $D^{(*)} \bar{D}^{(*)}$ thresholds [22], such as $X(3872)[23,24]$ and the charged charmonium $Z_{c}(3900)$ [25]. In the bottom quark sector, charged twin resonances $Z_{b}$ and $Z_{b}^{(\prime)}$ have been studied as the $B^{(*)} \bar{B}^{*}$ molecules [26].

The hadronic molecules are dynamically generated by the hadron-hadron interaction. Therefore, the properties of the interaction are important in producing those structures. In the charm quark sector, the SU(4) flavor symmetric interaction has been applied, and has also been used in the hidden-charm molecules [6-8]. However, the large mass of the charm quark would break the SU(4) flavor symmetry.

In the heavy flavor sector, the large mass of the heavy quarks introduces the heavy quark spin symmetry [27-29]. This symmetry appears because the suppression of the spin-dependent interaction between a heavy quark and a light degrees of freedom, a light quark and a gluon. It manifests the mass

^e-mail: yasuhiro.yamaguchi@ge.infn.it 
Table 1. Various channels of the $\bar{D}^{(*)} \Lambda_{\mathrm{c}}$ and $\bar{D}^{(*)} \Sigma_{\mathrm{c}}^{(*)}$ states for the given total angular momentum and parity $J^{P}$. In each channel, the total spin $S$ and orbital angular momentum $L$ are represented as ${ }^{2 S+1} L$.

\begin{tabular}{c|l}
\hline$J^{P}$ & Channels \\
\hline $3 / 2^{-}$ & $\bar{D} \Lambda_{\mathrm{c}}\left({ }^{2} D\right), \bar{D}^{*} \Lambda_{\mathrm{c}}\left({ }^{4} S,{ }^{2} D,{ }^{4} D\right), \bar{D} \Sigma_{\mathrm{c}}\left({ }^{2} D\right), \bar{D} \Sigma_{\mathrm{c}}^{*}\left({ }^{4} S,{ }^{4} D\right), \bar{D}^{*} \Sigma_{\mathrm{c}}\left({ }^{4} S,{ }^{2} D,{ }^{4} D\right)$, \\
& $\bar{D}^{*} \Sigma_{\mathrm{c}}^{*}\left({ }^{4} S,{ }^{2} D,{ }^{4} D,{ }^{6} D,{ }^{6} G\right)$ \\
$3 / 2^{+}$ & $\bar{D} \Lambda_{\mathrm{c}}\left({ }^{2} P\right), \bar{D}^{*} \Lambda_{\mathrm{c}}\left({ }^{2} P,{ }^{4} P,{ }^{4} F\right), \bar{D} \Sigma_{\mathrm{c}}\left({ }^{2} P\right), \bar{D} \Sigma_{\mathrm{c}}^{*}\left({ }^{4} P,{ }^{4} F\right), \bar{D}^{*} \Sigma_{\mathrm{c}}\left({ }^{2} P,{ }^{4} P,{ }^{4} F\right)$, \\
& $\bar{D}^{*} \Sigma_{\mathrm{c}}^{*}\left({ }^{2} P,{ }^{4} P,{ }^{6} P,{ }^{4} F,{ }^{6} F\right)$ \\
$5 / 2^{-}$ & $\bar{D} \Lambda_{\mathrm{c}}\left({ }^{2} D\right), \bar{D}^{*} \Lambda_{\mathrm{c}}\left({ }^{2} D,{ }^{4} D,{ }^{4} G\right), \bar{D} \Sigma_{\mathrm{c}}\left({ }^{2} D\right), \bar{D} \Sigma_{\mathrm{c}}^{*}\left({ }^{4} D,{ }^{4} G\right), \bar{D}^{*} \Sigma_{\mathrm{c}}\left({ }^{2} D,{ }^{4} D,{ }^{4} G\right)$, \\
& $\bar{D}^{*} \Sigma_{\mathrm{c}}^{*}\left({ }^{6} S,{ }^{2} D,{ }^{4} D,{ }^{6} D,{ }^{4} G,{ }^{6} G\right)$ \\
$5 / 2^{+}$ & $\left.\bar{D} \Lambda_{\mathrm{c}}\left({ }^{2} F\right), \bar{D}^{*} \Lambda_{\mathrm{c}} \mathrm{c}{ }^{4} P,{ }^{2} F,{ }^{4} F\right), \bar{D} \Sigma_{\mathrm{c}}\left({ }^{2} F\right), \bar{D} \Sigma_{\mathrm{c}}^{*}\left({ }^{4} P,{ }^{4} F\right), \bar{D}^{*} \Sigma_{\mathrm{c}}\left({ }^{4} P,{ }^{2} F,{ }^{4} F\right)$, \\
& $\bar{D}^{*} \Sigma_{\mathrm{c}}^{*}\left({ }^{4} P,{ }^{6} P,{ }^{2} F,{ }^{4} F,{ }^{6} F,{ }^{6} H\right)$ \\
\hline
\end{tabular}

degeneracy of the heavy hadrons with different total spin, such as $D$ and $D^{*}$ mesons $\left(\Delta m_{D D^{*}} \sim 140\right.$ $\mathrm{MeV})$ and $\Sigma_{c}$ and $\Sigma_{c}^{*}$ baryons $\left(\Delta m_{\Sigma_{\mathrm{c}} \Sigma_{\mathrm{c}}^{*}} \sim 65 \mathrm{MeV}\right)$. Therefore, the mass degeneracy induces a coupledchannel system. In the hidden-charm molecules formed by the $\bar{D}^{(*)}$ meson and the $\Sigma_{\mathrm{c}}^{(*)}$ baryons, the $\bar{D} \Sigma_{\mathrm{c}}, \bar{D} \Sigma_{\mathrm{c}}^{*}, \bar{D}^{*} \Sigma_{\mathrm{c}}$ and $\bar{D}^{*} \Sigma_{\mathrm{c}}^{*}$ channels will be coupled.

Moreover, we cannot ignore the $\bar{D}^{(*)} \Lambda_{\mathrm{c}}$ channel which was not considered in the early works of the hidden-charm pentaquarks $[7,8,13,14]$. The $\bar{D}^{*} \Lambda_{\mathrm{c}}$ threshold is $25 \mathrm{MeV}$ below the $\bar{D} \Sigma_{\mathrm{c}}$ threshold, and therefore, the $\bar{D}^{*} \Lambda_{\mathrm{c}}$ channel is not irrelevant.

In the $\bar{D}^{(*)} \Lambda_{\mathrm{c}}-\bar{D}^{(*)} \Sigma_{\mathrm{c}}^{(*)}$ system, the tensor force mixing $S$-wave and $D$-wave components would be important. In the $\bar{D} N-\bar{D}^{*} N$ systems, the $\bar{D}-\bar{D}^{*}$ mixing enhances the effect of the one pion exchange potential (OPEP) having the tensor force producing the strong attraction [30-35]. We expect that this mixing is also helpful to produce the attraction in the hidden-charm molecules.

On the basis of the above discussions, we consider the coupled-channel systems of $\bar{D}^{(*)} \Lambda_{c}-\bar{D}^{(*)} \Sigma_{c}^{(*)}$ including states with larger orbital angular momentum, namely $D$-wave and $G$-wave for the negative parity state, and $F$-wave and $H$-wave for the positive parity state, as summarized in Table 1 . This full-channel coupling has never been considered so far. The interaction is obtained by the one meson exchange potential that respects the heavy quark spin symmetry. The bound and resonant states in $I\left(J^{P}\right)=1 / 2\left(3 / 2^{ \pm}\right)$and $1 / 2\left(5 / 2^{ \pm}\right)$are studied by solving the coupled-channel Schrödinger equations.

\section{Interactions}

We employ the meson exchange potential as the interaction between the heavy meson and the heavy baryon. The meson exchange potential is obtained by the Lagrangians satisfying the heavy quark and chiral symmetries. The Lagrangians for a heavy meson and a light meson are given [29, 36-39] as

$$
\begin{aligned}
\mathcal{L}_{\pi H H} & =g_{\pi} \operatorname{Tr}\left[H_{b} \gamma_{\mu} \gamma_{5} A_{b a}^{\mu} \bar{H}_{a}\right], \quad \mathcal{L}_{\sigma H H}=g_{s} \operatorname{Tr}\left[H_{a} \sigma \bar{H}_{a}\right], \\
\mathcal{L}_{v H H} & =-i \beta \operatorname{Tr}\left[H_{b} v^{\mu}\left(\rho_{\mu}\right)_{b a} \bar{H}_{a}\right]+i \lambda \operatorname{Tr}\left[H_{b} \sigma^{\mu v} F_{\mu \nu}(\rho)_{b a} \bar{H}_{a}\right],
\end{aligned}
$$

for the pion $(\pi)$, sigma meson $(\sigma)$, and vector $\left(\rho\right.$ and $\omega$ ) meson $(v) . v^{\mu}$ is a four-velocity of a heavy quark. The heavy meson field constructed by the pseudoscalar meson $P$ and vector meson $P^{*}$ are represented [29, 36-39] by

$$
H_{a}=\frac{1+p}{2}\left[P_{a \mu}^{*} \gamma^{\mu}-P_{a} \gamma_{5}\right], \quad \bar{H}_{a}=\gamma_{0} H_{a}^{\dagger} \gamma_{0},
$$


Table 2. Masses of the exchanged mesons and coupling constants of the interaction Lagrangians for the heavy mesons and heavy baryons $[15,32,40]$.

\begin{tabular}{cccc}
\hline & $m_{\alpha}[\mathrm{MeV}]$ & Meson & Baryon \\
\hline$\pi$ & 137.27 & $g_{\pi}=0.59$ & $g_{1}=(3 / \sqrt{8}) g_{4}=1.0$ \\
$\rho$ & 769.9 & $\beta=0.9$ & $\beta_{S}=-2 \beta_{B}=12.0 / g_{V}$ \\
& & $\lambda=0.59\left[\mathrm{GeV}^{-1}\right]$ & $\lambda_{S}=-2 \sqrt{2} \lambda_{I}=19.2 / g_{V}\left[\mathrm{GeV}^{-1}\right]$ \\
$\omega$ & 781.94 & $\beta=0.9$ & $\beta_{S}=-2 \beta_{B}=12.0 / g_{V}$ \\
& & $\lambda=0.59\left[\mathrm{GeV}^{-1}\right]$ & $\lambda_{S}=-2 \sqrt{2} \lambda_{I}=19.2 / g_{V}\left[\mathrm{GeV}^{-1}\right]$ \\
$\sigma$ & 550.0 & $g_{S}=-0.76$ & $\ell_{S}=-2 \ell_{B}=7.30$ \\
\hline
\end{tabular}

where the subscripts $a, b$ are for the light flavor. $A_{\mu}$ and $F_{\mu v}(\rho)$ are the axial vector current and the field tensor given by

$$
A_{\mu}=\frac{i}{2}\left(\xi^{\dagger} \partial_{\mu} \xi-\xi \partial_{\mu} \xi^{\dagger}\right), \quad F_{\mu v}(\rho)=\partial_{\mu} \rho_{v}-\partial_{v} \rho_{\mu},
$$

where $\xi=\exp \left(i \hat{\pi} / 2 f_{\pi}\right)$ with the pion decay constant $f_{\pi}=92.3 \mathrm{MeV}$.

The Lagrangians for a heavy baryon and a light meson $[38,40]$ are given by

$$
\begin{aligned}
& \mathcal{L}_{\pi B B}=\frac{3}{2} g_{1} i v_{\kappa} \epsilon^{\mu \nu \lambda \kappa} \operatorname{tr}\left[\bar{S}_{\mu} A_{v} S_{\lambda}\right]+g_{4} \operatorname{tr}\left[\bar{S}^{\mu} A_{\mu} B_{\overline{3}}\right]+\text { H.c. }, \quad \mathcal{L}_{\sigma B B}=\ell_{B} \operatorname{tr}\left[\bar{B}_{\overline{3}} \sigma B_{\overline{3}}\right]+\ell_{S} \operatorname{tr}\left[\bar{S}_{\mu} \sigma S^{\mu}\right], \\
& \mathcal{L}_{v B B}=-i \beta_{B} \operatorname{tr}\left[\bar{B}_{\overline{3}} v^{\mu} \rho_{\mu} B_{\overline{3}}\right]-i \beta_{S} \operatorname{tr}\left[\bar{S}_{\mu} v^{\alpha} \rho_{\alpha} S^{\mu}\right]+\lambda_{S} \operatorname{tr}\left[\bar{S}_{\mu} F^{\mu \nu} S_{v}\right]+i \lambda_{I} \epsilon^{\mu \nu \lambda \kappa} v_{\mu} \operatorname{tr}\left[\bar{S}_{v} F_{\lambda_{k}} B_{\overline{3}}\right]+\text { H.c. . }
\end{aligned}
$$

The superfield $S_{\mu}$ for $\Sigma_{Q}^{(*)}$ is represented by

$$
S_{\mu}=B_{6 \mu}^{*}+\frac{\delta}{\sqrt{3}}\left(\gamma_{\mu}+v_{\mu}\right) \gamma_{5} B_{6}, \quad \bar{S}_{\mu}=\gamma_{0} S_{\mu}^{\dagger} \gamma_{0} .
$$

The phase factor is chosen by $\delta=-1$ as discussed in Ref. [40]. The coupling constants of the Lagrangians are summarized in Table 2.

From the effective Lagrangians introduced above, we obtain the meson exchange potentials as

$$
\begin{aligned}
& V_{\pi}^{i j}(r)=G_{\pi}^{i j}\left[\vec{O}_{1}^{i} \cdot \vec{O}_{2}^{j} C\left(r ; m_{\pi}\right)+S_{O_{1}^{i} O_{2}^{j}}(\hat{r}) T\left(r ; m_{\pi}\right)\right], \quad V_{\sigma}^{i j}(r)=G_{\sigma}^{i j} C\left(r ; m_{\sigma}\right), \\
& V_{v}^{i j}(r)=G_{v}^{i j} C\left(r ; m_{v}\right)+F_{v}^{i j}\left[-2 \vec{O}_{1}^{i} \cdot \vec{O}_{2}^{j} C\left(r ; m_{v}\right)+S_{O_{1}^{i} O_{2}^{j}}(\hat{r}) T\left(r ; m_{v}\right)\right] .
\end{aligned}
$$

$i$ and $j$ stand for the indices of the channels. $G_{\alpha}^{i j}(\alpha=\pi, \rho, \omega, \sigma)$ is the constant of the $(i, j)$ component given by the coupling constants of the Lagrangians. $O_{1}^{i}$ and $O_{2}^{j}$ are the (transition) spin operator of the heavy meson and heavy baryon vertices, respectively [31-33, 40]. $S_{O_{1}^{i} O_{2}^{j}}(\hat{r})$ is the tensor operator $S_{O_{1}^{i} O_{2}^{j}}(\hat{r})=3 \vec{O}_{1}^{i} \cdot \hat{r} \vec{O}_{2}^{j} \cdot \hat{r}-\vec{O}_{1}^{i} \cdot \vec{O}_{2}^{j}$. The potential for the isovector mesons, $\pi$ and $\rho$, is multiplied by the isospin factor, $\sqrt{6}$ for $\bar{D}^{(*)} \Lambda_{\mathrm{c}}-\bar{D}^{(*)} \Sigma_{\mathrm{c}}^{(*)}$ and -2 for $\bar{D}^{(*)} \Sigma_{\mathrm{c}}^{(*)}-\bar{D}^{(*)} \Sigma_{\mathrm{c}}^{(*)}$ with $I=1 / 2$. The functions $C\left(r ; m_{\alpha}\right)$ and $T\left(r ; m_{\alpha}\right)$ are given by

$$
\begin{aligned}
& C\left(r ; m_{\alpha}\right)=\int \frac{d^{3} q}{(2 \pi)^{3}} \frac{m_{\alpha}^{2}}{\vec{q}^{2}+m_{\alpha}^{2}} e^{i \vec{q} \cdot \vec{r}} F_{\alpha}(\Lambda, \vec{q}), \\
& S_{O_{1}^{i} O_{2}^{j}}(\hat{r}) T\left(r ; m_{\alpha}\right)=\int \frac{d^{3} q}{(2 \pi)^{3}} \frac{-\vec{q}^{2}}{\vec{q}^{2}+m_{\alpha}^{2}} S_{O_{1}^{i} O_{2}^{j}}(\hat{q}) e^{i \vec{q} \cdot \vec{r}} F_{\alpha}(\Lambda, \vec{q}),
\end{aligned}
$$


(i) $I\left(J^{P}\right)=1 / 2\left(3 / 2^{-}\right)$

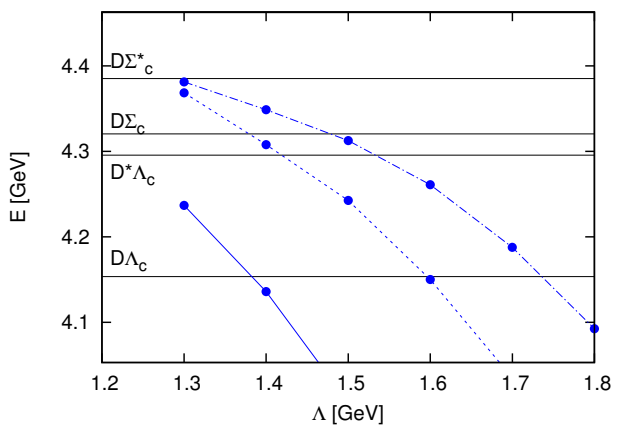

(iii) $I\left(J^{P}\right)=1 / 2\left(5 / 2^{-}\right)$

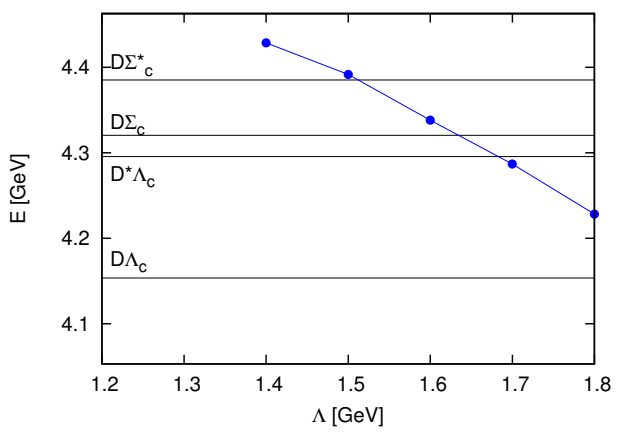

(ii) $I\left(J^{P}\right)=1 / 2\left(3 / 2^{+}\right)$

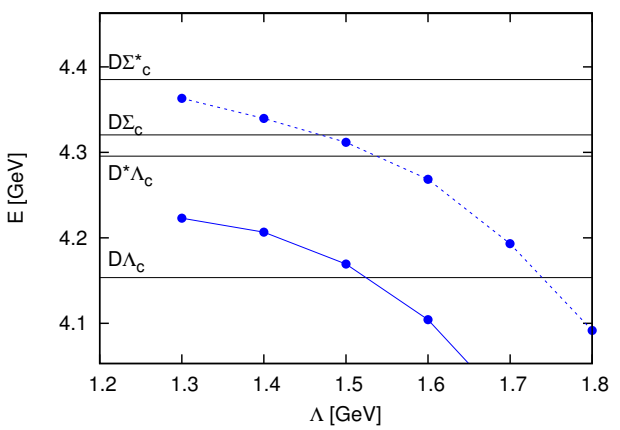

(iv) $I\left(J^{P}\right)=1 / 2\left(5 / 2^{+}\right)$

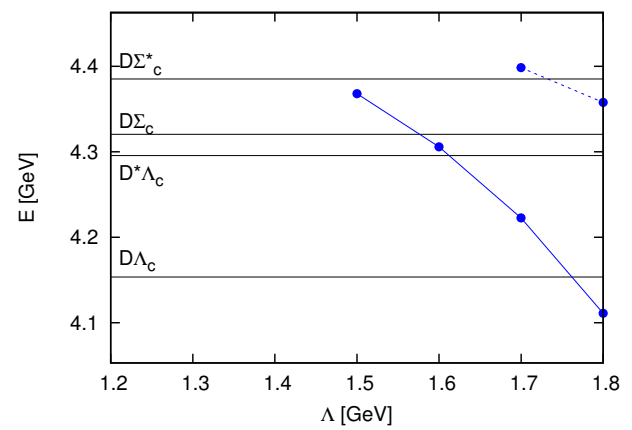

Figure 1. Cutoff $\Lambda$ dependence of the obtained energies $E$ in $J^{P}=3 / 2^{ \pm}$and $5 / 2^{ \pm}$and isospin $I=1 / 2$ from Ref. [15]. The black horizontal lines show the meson-baryon thresholds $\left(\bar{D}^{(*)} \Lambda_{\mathrm{c}}\right.$ and $\left.\bar{D}^{(*)} \Sigma_{\mathrm{c}}^{(*)}\right)$.

with the dipole-type form factor $F(\Lambda, \vec{q})$ for spatially extended hadrons [14, 31-33, 40]

$$
F_{\alpha}(\Lambda, \vec{q})=\left(\frac{\Lambda^{2}-m_{\alpha}^{2}}{\Lambda^{2}+\vec{q}^{2}}\right)^{2},
$$

with the cutoff parameters $\Lambda$.

\section{Numerical results}

We solve the Schrödinger equations with the meson exchange potential between the heavy meson and the heavy baryon for the coupled-channels. The molecular states of $\bar{D}^{(*)} \Lambda_{\mathrm{c}}^{(*)}-\bar{D}^{(*)} \Sigma^{(*)}$ with $J^{P}=3 / 2^{ \pm}, 5 / 2^{ \pm}$and isospin $I=1 / 2$ are studied. The obtained energies with the cutoff dependence are shown in Fig. 1. The cutoff parameter $\Lambda$ is determined to reproduce the observed mass of the hidden-charm pentaquarks. We then use the spectrum of the narrow resonance $P_{\mathrm{c}}^{+}(4450)$. We find that the state appearing near $P_{\mathrm{c}}^{+}(4450)$, is the $J^{P}=5 / 2^{-}$state with the resonance energy $4428.6 \mathrm{MeV}$ in $\Lambda=1400$ in our calculation. Therefore, the cutoff $\Lambda$ is chosen by $\Lambda=1400$. We conclude that the $J^{P}$ assignment of $P_{\mathrm{c}}^{+}(4450)$ is $J^{P}=5 / 2^{-}$. In according to the analysis of the LHCb collaboration [1], the $J$ of the observed pentaquarks are $3 / 2$ and $5 / 2$, and these states have the opposite parity. Therefore, 
Table 3. Obtained masses with full channel coupling (Full), without $\bar{D}^{(*)} \Lambda_{\mathrm{c}}\left(\mathrm{w} / \mathrm{o} \bar{D}^{(*)} \Lambda_{\mathrm{c}}\right.$ ) and without large orbital angular momentum $\ell$ (w/o $\ell>0$ or w/o $\ell>1$ ) in $\Lambda=1400 \mathrm{MeV}$ from Ref. [15].

\begin{tabular}{ccl|ccl}
\hline$J^{P}$ & Channels & Mass $[\mathrm{MeV}]$ & $J^{P}$ & Channels & Mass $[\mathrm{MeV}]$ \\
\hline $3 / 2^{-}$ & Full & $4136.0,4307.9,4348.7$ & $3 / 2^{+}$ & Full & $4206.7,4339.7$ \\
& w/o $\bar{D}^{(*)} \Lambda_{\mathrm{c}}$ & $4278.4,4400.4$ & & w/o $\bar{D}^{(*)} \Lambda_{\mathrm{c}}$ & - \\
w/o $\ell>0$ & $4220.4,4376.6$ & & w/o $\ell>1$ & 4275.3 \\
\hline $5 / 2^{-}$ & Full & 4428.6 & $5 / 2^{+}$ & Full & - \\
& w/o $\bar{D}^{(*)} \Lambda_{\mathrm{c}}$ & - & & w/o $\bar{D}^{(*)} \Lambda_{\mathrm{c}}$ & - \\
w/o $\ell>0$ & - & & w/o $\ell>0$ & - \\
\hline
\end{tabular}

the $J^{P}$ of the $P_{\mathrm{c}}^{+}(4380)$ will be $3 / 2^{+}$. In Fig. 1 , the upper state of $J^{P}=3 / 2^{+}$has the mass, 4339.7 $\mathrm{MeV}$, which is near the mass of $P_{\mathrm{c}}^{+}(4380)$. Therefore, this obtained state of $J^{P}=3 / 2^{+}$corresponds to $P_{\mathrm{c}}^{+}(4380)$.

In addition, new resonances are found in $\Lambda=1400 \mathrm{MeV}$. As shown in Fig. 1, we obtain three states in $J^{P}=3 / 2^{-}$, whose masses are $4136.0 \mathrm{MeV}, 4307.9 \mathrm{MeV}$ and $4348.7 \mathrm{MeV}$, respectively. In the $J^{P}=3 / 2^{+}$state, we find two states, where the upper state corresponds to $P_{\mathrm{c}}^{+}(4380)$ as discussed above, and the lower state is new one with the mass, $4206.7 \mathrm{MeV}$.

Finally, we discuss the effects of the channel couplings in the meson-baryon molecules. In the obtained results shown in Fig. 1, the full-channel coupling of $\bar{D}^{(*)} \Lambda_{\mathrm{c}}-\bar{D}^{(*)} \Sigma_{\mathrm{c}}^{(*)}$ is considered. However, the mass spectra are changed when the some of the channels are ignored. In Table 3 , we compare the results with full channel coupling, without $\bar{D}^{(*)} \Lambda_{\mathrm{c}}$, and without large orbital angular momentum $\ell>0$ $(\ell>1)$ for the negative (positive) parity. We find that the masses increase by tens of $\mathrm{MeV}$ and some of states disappear when the $\bar{D}^{(*)} \Lambda_{\mathrm{c}}$ channel or the states with large orbital angular momentum are ignored. Specifically the $J^{P}=5 / 2^{-}$state which corresponds to $P_{\mathrm{c}}^{+}(4450)$ disappears when the full channel coupling calculation is not carried out.

\section{Summary}

We studied the hidden-charm pentaquarks as meson-baryon molecules, $\bar{D}^{(*)} \Lambda_{\mathrm{c}}-\bar{D}^{(*)} \Sigma_{\mathrm{c}}$. We considered the channel coupling analysis with $\bar{D}^{(*)} \Sigma_{\mathrm{c}}^{(*)}$ manifested by the heavy quark spin symmetry, $\bar{D}^{(*)} \Lambda_{\mathrm{c}}$ near the $\bar{D}^{(*)} \Sigma_{\mathrm{c}}^{(*)}$ thresholds, and the states with larger orbital angular momentum mixed by the tensor force. The analysis of this molecular systems with $\bar{D}^{(*)} \Lambda_{\mathrm{c}}-\bar{D}^{(*)} \Sigma_{\mathrm{c}}^{(*)}$ had not been performed so far. As for the meson-baryon interaction, the meson exchange potential was employed which was obtained by the effective Lagrangians that respects the heavy quark and chiral symmetries. By solving the coupledchannel Schrödinger equations, we studied the bound and resonant states in $I\left(J^{P}\right)=1 / 2\left(3 / 2^{ \pm}\right)$and $1 / 2\left(5 / 2^{ \pm}\right)$with $I=1 / 2$. We concluded that the $J^{P}$ assignments of $P_{\mathrm{c}}^{+}(4380)$ and $P_{\mathrm{c}}^{+}(4450)$ are $3 / 2^{+}$ and $5 / 2^{-}$, respectively. We also found new states in $J^{P}=3 / 2^{ \pm}$. In this calculations, we found that the coupling to the $\bar{D}^{(*)} \Lambda_{\mathrm{c}}$ channel and to the state with large orbital angular momentum is not ignored, which produced the attraction.

\section{Acknowledgments}

This work was supported in part by the INFN Fellowship Programme.

\section{References}

[1] R. Aaij et al. [LHCb Collaboration], Phys. Rev. Lett. 115, 072001 (2015). 
[2] W. L. Wang, F. Huang, Z. Y. Zhang and B. S. Zou, Phys. Rev. C 84, 015203 (2011).

[3] S. G. Yuan, K. W. Wei, J. He, H. S. Xu and B. S. Zou, Eur. Phys. J. A 48, 61 (2012).

[4] G. N. Li, X. G. He and M. He, JHEP 1512, 128 (2015).

[5] G. Yang and J. Ping, arXiv:1511.09053 [hep-ph].

[6] J. Hofmann and M. F. M. Lutz, Nucl. Phys. A 763, 90 (2005).

[7] J. J. Wu, R. Molina, E. Oset and B. S. Zou, Phys. Rev. Lett. 105, 232001 (2010).

[8] J. J. Wu, R. Molina, E. Oset and B. S. Zou, Phys. Rev. C 84, 015202 (2011).

[9] C. Garcia-Recio, J. Nieves, O. Romanets, L. L. Salcedo and L. Tolos, Phys. Rev. D 87, 074034 (2013).

[10] C. W. Xiao, J. Nieves and E. Oset, Phys. Rev. D 88, 056012 (2013).

[11] M. Karliner and J. L. Rosner, Phys. Rev. Lett. 115, no. 12, 122001 (2015).

[12] T. Uchino, W. H. Liang and E. Oset, Eur. Phys. J. A 52, no. 3, 43 (2016).

[13] R. Chen, X. Liu and S. L. Zhu, Nucl. Phys. A 954, 406 (2016).

[14] Y. Shimizu, D. Suenaga and M. Harada, Phys. Rev. D 93, no. 11, 114003 (2016).

[15] Y. Yamaguchi and E. Santopinto, arXiv:1606.08330 [hep-ph].

[16] F. K. Guo, U. G. Meißner, W. Wang and Z. Yang, Phys. Rev. D 92, 071502 (2015).

[17] X. H. Liu, Q. Wang and Q. Zhao, Phys. Lett. B 757, 231 (2016).

[18] F. K. Guo, U. G. Meißner, J. Nieves and Z. Yang, arXiv:1605.05113 [hep-ph].

[19] E. Oset and A. Ramos, Nucl. Phys. A 635, 99 (1998).

[20] B. Krippa, Phys. Rev. C 58, 1333 (1998).

[21] T. Hyodo and D. Jido, Prog. Part. Nucl. Phys. 67, 55 (2012).

[22] N. Brambilla et al., Eur. Phys. J. C 71 (2011) 1534.

[23] S. K. Choi et al. [Belle Collaboration], Phys. Rev. Lett. 91, 262001 (2003).

[24] E. S. Swanson, Phys. Rept. 429, 243 (2006).

[25] M. Ablikim et al. [BESIII Collaboration], Phys. Rev. Lett. 110, 252001 (2013).

[26] A. Bondar et al. [Belle Collaboration], Phys. Rev. Lett. 108, 122001 (2012).

[27] N. Isgur and M. B. Wise, Phys. Lett. B 232, 113 (1989).

[28] N. Isgur and M. B. Wise, Phys. Lett. B 237, 527 (1990).

[29] A. V. Manohar and M. B. Wise, Heavy quark physics (Camb. Monogr. Part. Phys. Nucl. Phys.

Cosmol. 10, 2000) 1.

[30] T. D. Cohen, P. M. Hohler and R. F. Lebed, Phys. Rev. D 72, 074010 (2005).

[31] S. Yasui and K. Sudoh, Phys. Rev. D 80, 034008 (2009).

[32] Y. Yamaguchi, S. Ohkoda, S. Yasui and A. Hosaka, Phys. Rev. D 84, 014032 (2011).

[33] Y. Yamaguchi, S. Ohkoda, S. Yasui and A. Hosaka, Phys. Rev. D 85, 054003 (2012).

[34] Y. Yamaguchi, S. Ohkoda, S. Yasui and A. Hosaka, Phys. Rev. D 87, 074019 (2013).

[35] A. Hosaka, T. Hyodo, K. Sudoh, Y. Yamaguchi and S. Yasui, arXiv:1606.08685 [hep-ph].

[36] M. B. Wise, Phys. Rev. D 45, no. 7, R2188 (1992).

[37] A. F. Falk and M. E. Luke, Phys. Lett. B 292, 119 (1992).

[38] T. M. Yan, H. Y. Cheng, C. Y. Cheung, G. L. Lin, Y. C. Lin and H. L. Yu, Phys. Rev. D 46, 1148 (1992) Erratum: [Phys. Rev. D 55, 5851 (1997)].

[39] R. Casalbuoni, A. Deandrea, N. Di Bartolomeo, R. Gatto, F. Feruglio and G. Nardulli, Phys.

Rept. 281, 145 (1997).

[40] Y. R. Liu and M. Oka, Phys. Rev. D 85, 014015 (2012). 